\title{
CAP-Verdacht bei Älteren: Sono schlägt Röntgen
}

Hintergrund u. Fragestellung: Die ambulant erworbene Pneumonie (CAP) ist die häufigste Infektionskrankheit und im hohen Alter mit hoher Morbidität und Mortalität verbunden. Daher ist die frühzeitige Diagnostik unerlässlich, um rechtzeitig adäquat zu therapieren. Ist die Lungen-Sonografie dem konventionellen Röntgen-Thorax hier überlegen?

Patienten u. Methoden: Bei Patienten mit Verdacht auf CAP wurde in der Notaufnahme durch ausgebildete, erfahrene Ärzte eine Sonografien durchgeführt (SonoSite M-Turbo 3,5-

\section{Originalie}

Liu XL, Lian R, Tao YK et al. Lung ultrasonography: an effective way to diagnose community-acquired pneumonia. Emerg Med J. 2015 Jun;32(6):433-8.
$5 \mathrm{MHz}$ ). Voraussetzung für die Untersucher war, dass sie mindestens fünfzig Ultraschalluntersuchungen bei Pneumonie vorgenommen hatten, basierend auf einem 28-Stunden-Kurs. Es wurde immer nach einem klaren Muster vorgegangen, das dem FASTKonzept entspricht. Definitionen für infiltrative Nachweise, interstitielle Veränderungen, subpleurale Läsionen und pleuritische Anomalien wurden im Vorfeld festgelegt. Zudem wurde bei den Patienten ein konventioneller Röntgen-Thorax gemacht.

Ergebnisse: 223 Patienten wurden in die Studie aufgenommen und in der Notaufnahme bei V.a. CAP entsprechend untersucht. Das mittlere Alter war 72 Jahre. Im Vergleich zum konventionellen Röntgen lagen die Sensitivität und die Spezifität der Sonografie gegenüber dem Röntgen deutlich höher. Die Sensitivität der Sonografie lag bei $94,6 \%$ (vs. 77,7\%), die Spezifität bei 98,5\% (vs. 94,0\%) und die diagnostische Treffsicherheit bei 96,1\% (vs. 83,8\%).

Schlussfolgerung: Die Sonografie als eine nicht invasive und patientennahe Untersuchung bei Verdacht auf Pneumonie hat eine höhere Ergebnisqualität als der konventionelle RöntgenThorax.

\section{- Kommentar von Univ.- Prof. Dr. med. Hans Jürgen Heppner}

\section{Spezielle Schulungen und Abeitsprotokolle nötig}

Die patientennahe Sonografie in der Notaufnahme ist bei verschiedenen Krankheitsbildern bereits etabliert. Auch für die Diagnose des Lungenödems und zuverlässig in der Diagnostik des Pneumothorax wird sie verbreitet eingesetzt [1, 2]. Spezielle Schulungsmodule bereiten auf den Einsatz vor und liefern ein Arbeitsprotokoll [3]. Hier konnten die Autoren zeigen, dass die Sonografie dem konventionellen Röntgen in Bezug auf den CAP-Nachweis überlegen ist. Dies öffnet natürlich für das sehr empfindliche Gut der geriatrischen Patienten eine neue Option. So kann zeitnah befundet und die Diagnose mithilfe des Ultraschalls schonend und schnell gestellt werden, ohne dass die Patienten für weiterführende Untersuchungen in andere Abteilungen gebracht werden. Die Sonografie des älteren Patienten mit V.a. auf CAP ist vielversprechend, aber nicht ganz einfach, da sie eine Schulung voraussetzt. Dafür gilt es, Arbeitsprotokolle in den Kliniken zu implementieren.

\section{Literatur}

1. Liu XL, Lian R, Tao YK et al. Emerg Med J. 2015 Jun;32(6):433-8.

2. Ye X, Xiao H, Chen B, Zhang S. PLoS One. 2015 Jun 24;10(6):e0130066.

3. Röhrig S, Seibel A, Zechner PM et al. Anästhesiol Intensivmed Notfallmed Schmerzther 2011;46:772-80.

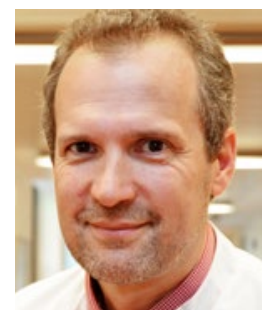

Univ.- Prof. Dr. med. Hans Jürgen Heppner, MHBA

Chefarzt Geriatrische Klinik und Tagesklinik Lehrstuhl für Geriatrie Universität Witten/Herdecke Dr.-Moeller-Straße 15, 58332 Schwelm hans.heppner@uni-wh.de

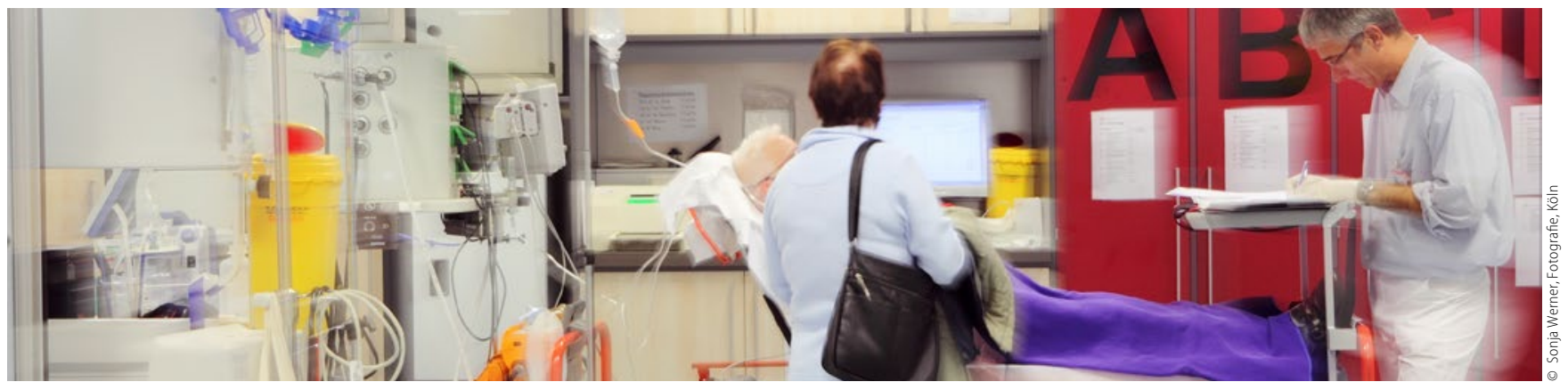

Mithilfe des Ultraschalls kann eine Pneumonie schonend und schnell bereits in der Notaufnahme diagnostiziert werden. 